\title{
Study on the Good Wind Characteristics over a Large Central City by Laser Radar Measurement
}

\author{
Li Yuandong, Quan Yong* \\ Email address: \\ 1832309@tongji.edu.cn (Li Yuandong), quanyong@tongji.edu.cn (Quan Yong) \\ ${ }^{*}$ Corresponding author
}

State Key Laboratory for Disaster Reduction in Civil Engineering, Tongji University, Shanghai, China

\section{To cite this article:}

Li Yuandong, Quan Yong. Study on the Good Wind Characteristics over a Large Central City by Laser Radar Measurement. Science Discovery. Vol. 9, No. 1, 2021, pp. 7-12. doi: 10.11648/j.sd.20210901.12

Received: December 29, 2020; Accepted: January 21, 2021; Published: January 28, 2021

\begin{abstract}
In recent years, the process of urbanization in my country has accelerated, the density of buildings in large cities has been increasing, and the height of buildings has also increased, resulting in an increasingly complex wind environment in the city center. An accurate and reasonable description of the characteristics of the wind field within the height of the atmospheric boundary layer in the city center is very important for determining the wind load of the high-rise building structure. This paper uses Windcube 100S Doppler laser wind measurement radar, taking Shanghai as an example, to carry out long-term actual measurement of good wind in the height range of 50m 1200m above Tongji University Wind Engineering Hall and obtain specific and detailed observation data. The time history of wind speed and direction in July and December 2019 is analyzed, and the average wind speed profile and average wind direction profile classified by incoming direction are studied, and the variation law of ground roughness index is obtained. The results show that there is no significant difference in the ground roughness index between different wind directions and seasons. The fitting values of ground roughness index in different directions for heights below 250m are greater than corresponding values for heights above $250 \mathrm{~m}$ in the same direction, and are greater than the load specification values under class $\mathrm{D}$ landforms.
\end{abstract}

Keywords: Field Measurement, Laser Radar, Long Period, Wind Speed Profile, Ground Roughness Index

\section{大城市中心良态风特性的激光雷达实测研究}

\author{
李远东, 全涌* \\ 土木工程防灾国家重点实验室, 同济大学, 上海, 中国 \\ 邮箱 \\ 1832309@tongji.edu.cn (李远东), quanyong@tongji.edu.cn(全涌)
}

摘要: 近年来我国城市化进程加快, 城市建筑密度不断增大, 建筑高度也日益增加, 由此造成城市中心风环境日益复杂的局面。准确 合理地描述大城市中心大气边界层风场特性, 对于确定高层建筑结构风荷载至关重要。本文利用Windcube $100 \mathrm{~S}$ 多普勒激光测风雷 达, 以上海市为例, 对同济大学上空 $50 \mathrm{~m}$ 1200m高度范围内的良态风进行了长期实测并得到具体详实的观测数据。分析 了2019年7月、12月期间的风速风向时程, 研究了按来流方向分类的平均风速剖面及平均风向剖面, 并得出地面粗糙度指 数变化的规律。结果表明: 不同风向不同季节所拟合出的地面粗䊁度指数无明显差别。250m以下高度不同方向的地面粗 糙度指数拟合值均大于同方向 $250 \mathrm{~m}$ 以上高度的对应值，且大于 $\mathrm{D}$ 类地貌下荷载规范值。夏季风向剖面曲线比冬季稳定; 高度较低区域风向剖面曲线比高度较高区域稳定; 风速较小区间的风向剖面曲线比风速较大区间稳定。

关键词: 现场实测, 激光雷达, 长周期, 风剖面, 地面粗糙度指数 


\section{1. 引言}

随着经济社会的发展和城市化进程的加快, 我国逐渐 形成了以超大型城市为中心的大型城市群, 如以北京为中 心的京津唐地区、以上海为中心的长三角地区和以深圳为 中心的粤港澳的湾区等。这些城市群内部建筑密度不断增 大, 建筑结构日益复杂, 建筑高度也逐渐提高, 现有荷载 规范已经不足以描述城市中心风场特性的实际情况。而合 理的描述城市地貌下大气边界层风场特性, 对于确定风敏 感结构的荷载, 对于结构风致振动的分析十分重要。因此, 以上问题的研究对于保证建筑工程结构的安全性和经济性, 对于高层建筑的建设和城市化进程的推进都具有重要意义。

尽管随着计算机计算能力的提高, 数值模拟等研究方 法逐渐受到重视, 但现场实测始终是研究风场特性的重要 手段。随着时代的发展, 现场实测的水平和精度也日益提 高。初期, 风场特性实测主要依靠气象手段。Durst基于大 量气象塔观测数据, 得到了平均风速大小随高度的变化关 系, 以及风速与平均时距之间的拟合公式, 为后人的研究 奠定了基础[1]; 傅抱璞等分析了南京八卦洲气象塔风速剖 面特点, 得出了粗糙度参数随风速增大而趋近于稳定值这 一基本规律 [2]。Cook于1997年通过英国气象站测得的风 速风向数据分析并比较了指数率和对数率风速剖面模型 [3]。王澈泉等基于台风“麦德姆”在城市地貌的高空实测风 场资料, 发现时距小, 平均风速大, 湍流度小, 但其变异 系数大; 时距大, 平均风速小, 湍流度大, 但其变异系数 小; 并对比指出, 当平均时距为 $5 \mathrm{~min}$ 时, 采用自相关函数 积分法得到的湍流积分尺度最为合理[4]。

随着建筑密度和高度不断增大, 城市建成面积也不断 扩大, 城市地貌变得更加复杂, 对实测的精度有了更高要 求。观测位置逐渐由郊外平坦地区转移到城市中心的高层 建筑顶部。申建红对青岛泽润广场 $113 \mathrm{~m}$ 高处风场进行了实 测, 发现在低频区域, 实测脉动风速的标准谱密度介于 Davenport谱和Kaimal谱之间, 高频区域的谱值则与典型风 谱有比较大差异 [5]。何浩博等对天津 117 大厦 $478 \mathrm{~m}$ 处的风 场实测数据进行处理, 显示该处脉动风速并没有呈现明显 的高斯概率分布 [6]; 顾明等利用上海环球金融中心 $494 \mathrm{~m}$ 高 度处风速采集仪的风速数据, 对城市风场的平均及脉动特 性进行了长期观测和系统研究 [7]; Q.S. Li等于2012年在 420m高层建筑顶部对路过香港的“文森特”台风进行了 18 小 时风场观测, 并进行了高层建筑结构风致震动分析 [8]。

雷达技术的发展对风场特性的现场实测具有重要意 义。因其测量方便, 不依赖高层建筑顶部, 可进行全高度 测量, 且数据质量切实可靠等特点, 甫一出现就受到研究 人员的重视。Rothermel (1998) 利用NASA推出的激光雷 达系统测得了从地面到 $30 \mathrm{~km}$ 高度范围的风场 [9]; 王乔乔 等 (2013) 采用Windcube激光雷达测风系统与测风塔测风 设备同步观测, 计算了各种统计量, 对风速、风向、标准 偏差和湍流强度进行了对比分析 [10]; 陈雯超等（2017） 利用不同测风仪器的同步测量数据, 比较分析了不同天气 条件下激光测风雷达的性能可靠性等[11]。都是雷达技术 在风场特性现场实测领域的应用与进步。
本文利用Windcube 100S多普勒激光测风雷达, 对上 海市密云路同济大学附近大气边界层内风场特性进行了 现场实测, 获得了典型大城市中心区域 $50 \mathrm{~m}$ 到 $1200 \mathrm{~m}$ 范围 内不同季节的良态强风长期观测数据, 研究了2019年夏季 和冬季的风速风向时程, 分析了不同观测时距对平均风速 的影响, 以及不同季节风速风向剖面特性。

\section{2. 实测方案}

\section{1. 观测环境}

本次实测将测风雷达安置在上海市同济大学四平 路校区风工程馆靠近密云路一侧空地上, 如图 1 所示。 实测现场周边环境颇为复杂。西侧北侧紧邻密云路内 环高架路, 东侧和南侧处于同济大学校内, 附近有同济大 学桥梁馆和生态实验楼等建筑。观测点周围 $500 \mathrm{~m}$ 内包含 大量 $100 \mathrm{~m}$ 及以上高度建筑。该位置地处五角场城市副中 心和四川北路商圈范围内, 周边有高层建筑群, 是典型的 城市中心地貌。

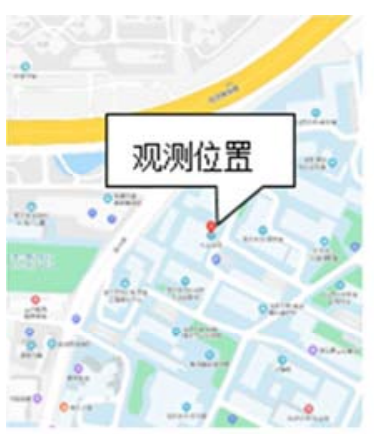

a 观测位置

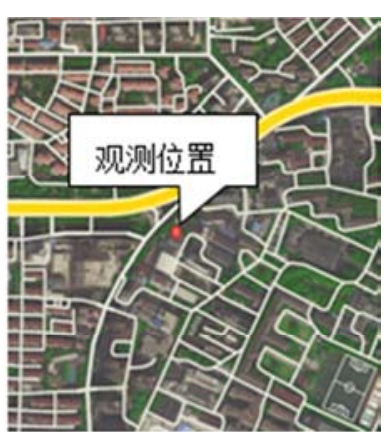

b 近场建筑环境
图1 观测位置及周边。

\section{2. 实测仪器设备}

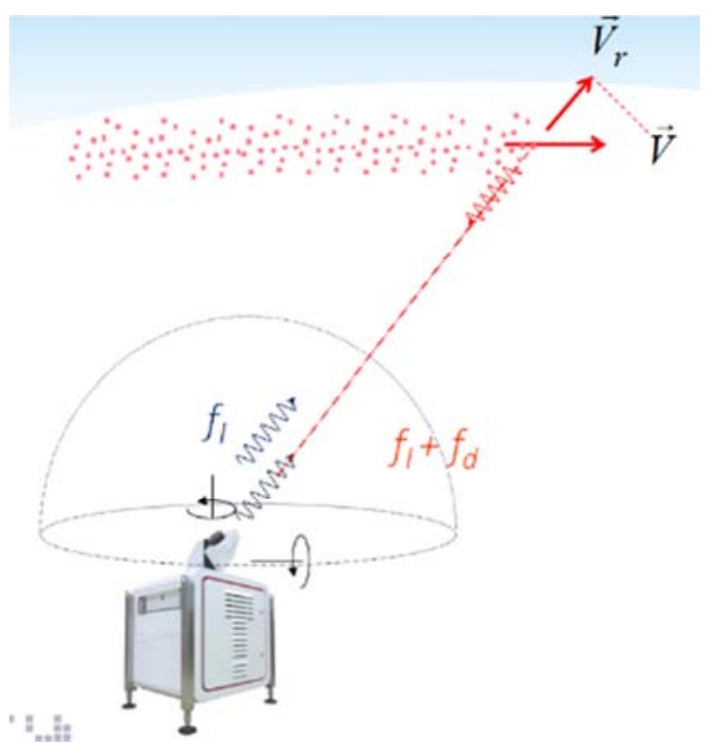

图2 Windcube 100S激光雷达工作原理。 
本次实测所用仪器为法国LEOSPHERE公司推出的 Windcube 100S版本多普勒激光测风雷达, 工作原理如图2 所示。激光雷达向大气发射一定波长的短光脉冲, 探测到 气溶胶的运动, 利用光的多普勒效应, 通过气溶胶反射的 激光脉冲获得沿激光方向的风速数据。

Windcube 100S多普勒激光测风雷达自动化程度高、 操作便捷, 所需维护工作量少, 对温度和湿度均无苛刻要 求, 对海洋环境和台风环境等不同使用条件均有很强的适 应性。相关性能参数如表 1 所示。

表1 Windcube 100S性能参数。

\begin{tabular}{ll}
\hline 参数类别 & 技术指标 \\
\hline 脉冲频率 & $10 \mathrm{kHz}$ \\
量程变化范围 & $50 \mathrm{~m}$ 至 $3500 \mathrm{~m}$ \\
方位角范围 & $0^{\circ}$ 至 $360^{\circ}$ \\
测量采集时间 & 0.5 至 10 秒 \\
分辨率 & $25 \mathrm{~m} 、 50 \mathrm{~m} 、 75 \mathrm{~m} 、 100 \mathrm{~m}$ \\
速度测量的精度 & $0.5 \mathrm{~m} / \mathrm{s}$ \\
径向风速的范围 & $-30 \mathrm{~m} / \mathrm{s}$ 至 $30 \mathrm{~m} / \mathrm{s}$ \\
尺寸和重量 & $995 \times 810 \times 1410 \mathrm{~mm}$ \\
温度范围 & $-25^{\circ} \mathrm{C}$ 至 $+45^{\circ} \mathrm{C}$ \\
重量 & $232 \mathrm{~kg}$ \\
\hline
\end{tabular}

本次实测采用其DBS模式, 如图4所示。该模式下雷 达以 $75^{\circ}$ 仰角做圆锥面扫描, 以 $90^{\circ}$ 仰角测得垂直风速, 直 接获取圆锥面上的径向风速 $\mathrm{Vr}$, 通过观测四个方向 $\left(0^{\circ}\right.$ 、 $90^{\circ} 、 180^{\circ}$ 和 $\left.270^{\circ}\right)$ 的径向风速和垂直风速, 利用三角函数 关系即可得到三维直角坐标系三个正交方向上的风速。扫 描模式如图4所示。

\section{3. 数据处理方法}

进行风特性分析之前, 需要将实测数据按一定长度进 行分割, 得到的子样本长度 $N=T * \mathrm{f}_{s}$, 其中 $\mathrm{f}_{s}$ 为采样频 率; $T$ 一般称作平均时距。研究表明, 平均风速与所取的 基本时距 $T$ 有关, 不同的平均时距 $T$ 对所得平均风速的影 响, 也是本文的重要研究内容之一。对于平均时距 $T$ 的取 值, 不同国家和地区的规范有一定差别, 一般 $T$ 取 $10 \mathrm{~min} \sim \mathrm{h}$ 较为稳定。

DBS风廓线模式下可直接同步测量纵向、横向和坚向三 个正交分量的风速时程, 分别对应 $\mathrm{x}, \mathrm{y}, \mathrm{z}$ 三轴方向上的风 速分量, 记为 $u_{x}(t) 、 u_{y}(t)$ 和 $u_{z}(t)$, 建立三维正交直角坐标系x-y-z, 设定 $\mathrm{x}$ 轴正向为北, $\mathrm{y}$ 轴正向为东, $\mathrm{z}$ 轴正向为垂直向上, 定 义 $\mathrm{x}$ 轴方向为 $0^{\circ}$, 沿顺时针依次增大, 如图 3 所示。

根据观测数据可以直接得出在三坐标轴上的风速, 即: 在正交坐标系下的三分量 $u_{x}(t) 、 u_{y}(t)$ 和 $u_{z}(t)$ 。在基本时距 $\mathrm{T}$ 内的水平平均风速 $U$ 、水平平均风向角 $\theta$ 由以下公式得到:

$$
\begin{aligned}
& U=\sqrt{{\overline{u_{x}(t)^{2}}}^{2}+{\overline{u_{y}(t)}}^{2}} \\
& \cos (\bar{\phi})=\overline{u_{x}(t)} / U ; \quad \sin (\bar{\phi})=\overline{u_{y}(t)} / U
\end{aligned}
$$

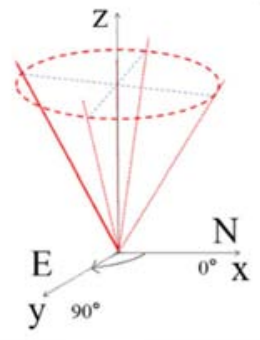

图3 风向角及空间坐标定义。

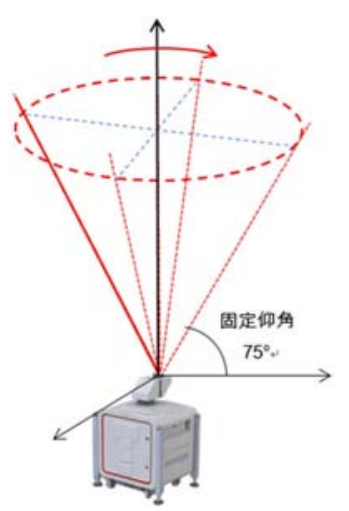

图4 DBS扫描模式。

\section{3. 数据处理结果}

\section{1. 平均风速}

本项目在2019年7月和12月进行了无间断现场实测， 得到了各一个月时长的夏季、冬季风速风向数据。经过对 异常点和无效点的处理, 并按照 $10 \mathrm{~min}$ 平均时距划分样本 后, 从中篎选出满足中性稳定条件的良态风, 共得到8289 个有效样本, 包括夏季 4175 个样本, 冬季 4114 个样本。
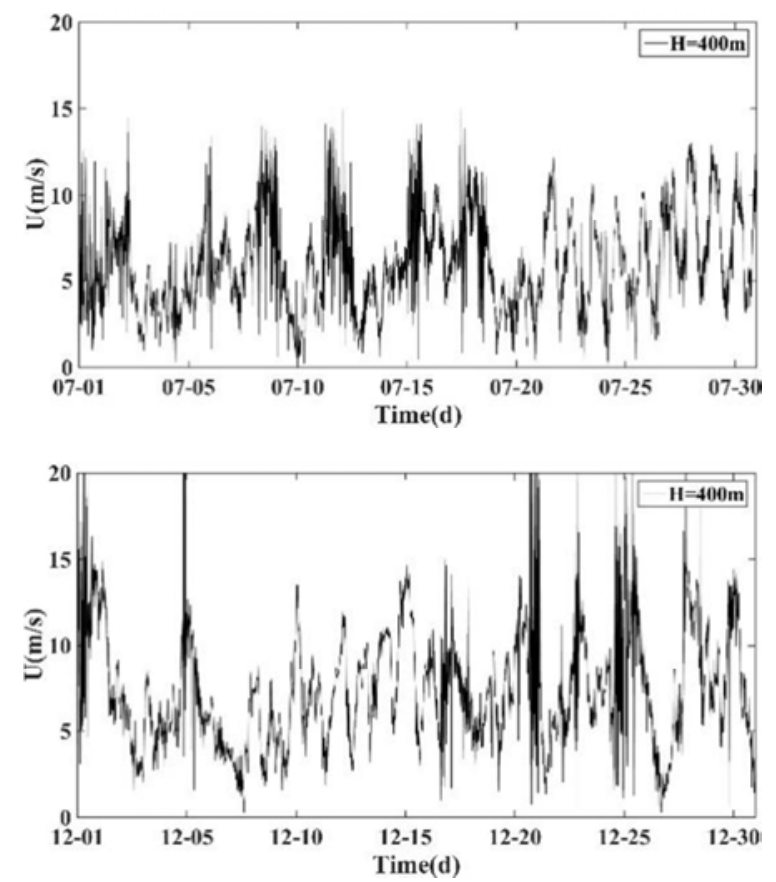

图5 7月、12月十分钟平均风速时程比较 $(\mathrm{H}=400 \mathrm{~m})$ 。 
图5展示了 2019年7月、12月观测数据在 $400 \mathrm{~m}$ 高度处 的十分钟平均风速时程。分析表明，随着观测时间的增加， 夏季和冬季的平均风速均有较大波动, 体现了良态风具有 脉动性。但与此同时, 平均风速又都在保持稳定水平上, 7月各天平均风速均不超过 $10 \mathrm{~m} \cdot \mathrm{s}^{-1}, 12$ 月各天平均风速更 高, 但有效样本中最大平均风速不超过 $20 \mathrm{~m} \cdot \mathrm{s}^{-1}$, 未达到强 风水平。

总体来看, 在大气边界层范围内, 平均风速随高度增 大而增加。空间上看, 各高度处的风速风向时程均呈现出 很强的脉动性; 时间上看, 不同季节平均风速的变化有一 定一致性，冬季风速会比夏季呈现更强的脉动性。

\section{2. 平均风速剖面}

分析主要来流方向上的十分钟平均风速样本, 以 50 $800 \mathrm{~m}$ 高度上的样本风速平均值为准, 按照 $3 \mathrm{~m} \cdot \mathrm{s}^{-1}$ 间隔 划分数据。得到各方向平均风速剖面图, 如图6图7所示。

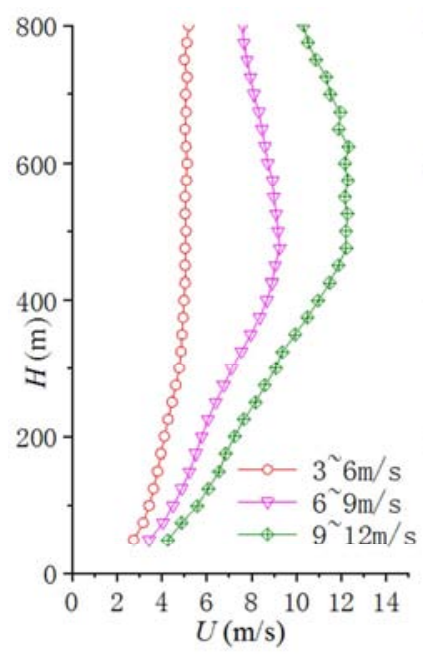

a $S$ 方向

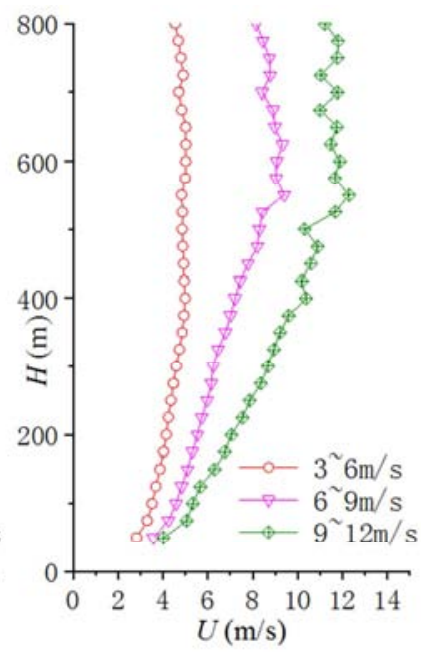

b SSE 方向
图67月主要来流方向平均风速剖面。

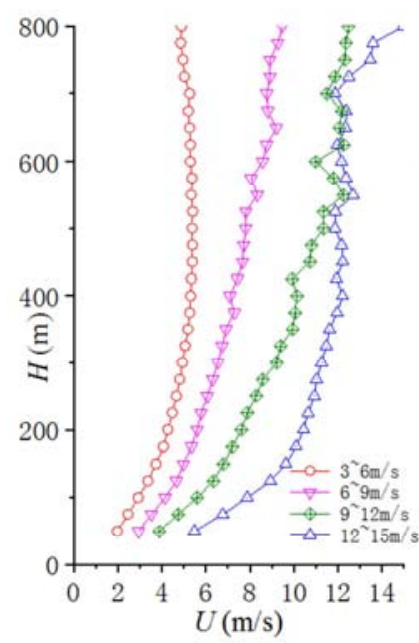

a $\mathrm{N}$ 方向

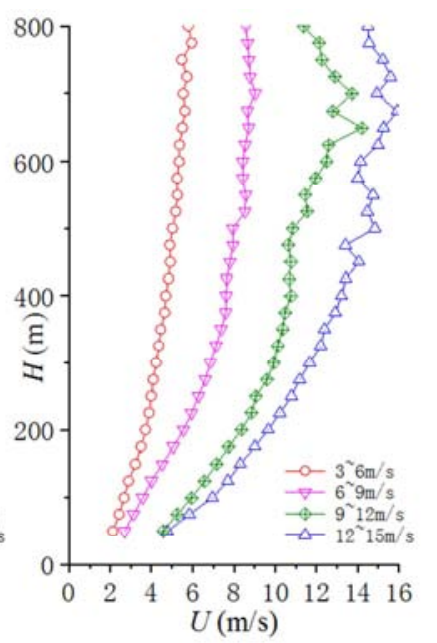

b NW 方向
对于7月份数据, 按照 100 米高度处, 即最低测量高度 处的来流风向, 将实测数据划分入 16 个风向角区间, 其有 效样本较多的来流风向主要有S、SSE、和SE方向, 分别 有 724 个、 518 个和 551 个个有效样本, 可见上海市 7 月来流 风向以南风和东南风为主; 对于 12 月份数据, 按同样标准 划分, 其有效样本较多的来流风向主要有 $\mathrm{N} 、 \mathrm{NNW}$ 和 $\mathrm{NW}$ 方向, 分别有 575 个、 495 个和 470 个有效样本, 来流风向 以北风和西北风为主。除主要风向外, 其他方向样本均较 少, 且变动频繁不稳定, 夏季相比于冬季, 来流更加集中 于主要方向, 风向更为稳定。

可见, 对于7月份样本, 风速较小时, 风速剖面无法 看出明显的规律性, 而且梯度风高度较低, 仅在300 400m 之间; 对于较大风速的样本, 风速剖面则有更为明显的规 律性, 不同方向上风速都随高度有显著变化, 梯度风高度 在500 600m之间。

12月份的样本变化趋势与7月份类似, 如图7所示。差 别在于: 冬季风剖面所显示的梯度风高度更低, 在低风速 区间在200 300m之间，在高风速区间也只有约400米; 且 冬季平均风速普遍较夏季为高, 在 $12 \sim 15 \mathrm{~m} \cdot \mathrm{s}^{-1}$ 高风速区间 仍有较多稳定样本。另外, 冬夏季样本均出现在 600 米以 上高度风剖面趋势变化不稳定的现象, 这种现象在高风速 区间的风剖面中尤为显著。可能是云层中的水汽和降水天 气对激光雷达的测量稳定性产生不良影响。

通过指数律经验公式拟合图6图7所示结果。

$$
\bar{U}(z)=\bar{U}_{s}\left(\frac{z}{z_{s}}\right)^{\alpha}
$$

其中, $\bar{U}(\mathrm{z})$ 和 $\mathrm{z}$ 为平均风速和观测点高度; $z_{s}$ 和 $\bar{U}_{s}$ 为 参考高度及其对应的平均风速; $\alpha$ 为地面粗䊁度指数。拟 合结果见表2。

表2 不同风速区间内的地面粗糙度指数。

\begin{tabular}{|c|c|c|c|c|c|}
\hline \multicolumn{2}{|c|}{ 参数 } & \multicolumn{4}{|l|}{ 值 } \\
\hline & & $3 \sim 6$ & $6 \sim 9$ & 9 12 & $12 \sim 15$ \\
\hline \multirow{3}{*}{$\alpha$} & NW & 0.21 & 0.34 & 0.38 & 0.33 \\
\hline & NNW & 0.18 & 0.37 & 0.42 & 0.39 \\
\hline & NWW & 0.23 & 0.29 & 0.33 & 0.35 \\
\hline \multirow{2}{*}{$\alpha$} & $\mathrm{S}$ & 0.23 & 0.35 & 0.43 & \\
\hline & SSE & 0.14 & 0.36 & 0.39 & \\
\hline
\end{tabular}

由表2可见, 冬夏季的地面粗糙度指数变化都是随着 风速增大而增大。在冬季高风速 $12 \sim 15 \mathrm{~m} \cdot \mathrm{s}^{-1}$ 区间, 地面粗 糙度指数趋于稳定, 相较9 $12 \mathrm{~m} \cdot \mathrm{s}^{-1}$ 区间无明显变化。由于 夏季高风速12 15 m $\cdot \mathrm{s}^{-1}$ 区间有效样本过少, 因此不作讨论。 以上结果是和 Tamura et al对多普勒声雷达长期观测数据 的分析结果一致的 [12]。分析表明, 大地边界层内, 在低 风速区间会受大气对流的热分层现象影响[13]; 只有风速 达到足够大, 其湍流特性对大气边界层的影响大于热分层 效应后, 边界层内地面粗䊁度指数才会趋于稳定。指数率 拟合效果如图8所示。

图7 12月主要来流方向平均风速剖面。 


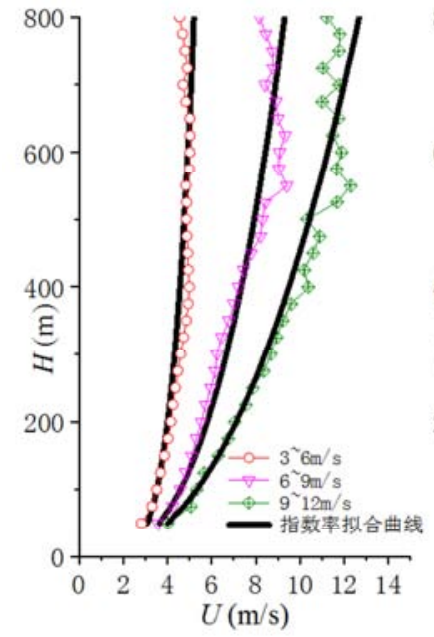

a SSE 方向

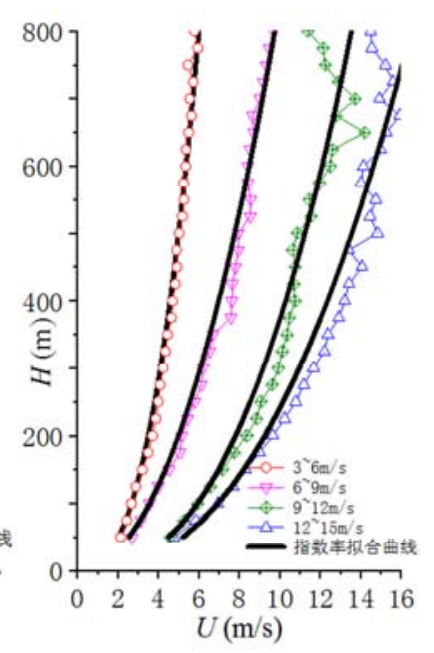

b NW 方向
图8 主要来流方向指数率拟合效果图。

不同方向风剖面曲线分别在200 300m高度附近出现 了拐点, 拐点两侧曲线有较大差异, 难以用同一函数曲线 拟合, 因此, 以冬季样本为例, 选择 $250 \mathrm{~m}$ 为拐点高度对 6 9 $\mathrm{m} \cdot \mathrm{s}^{-1}$ 区间下风剖面进行分段拟合，其结果见表 3 。

表3 地面粗粘度指数分段拟合结果 $\left(6 \mathrm{~m} \cdot \mathrm{s}^{-1} \sim 9 \mathrm{~m} \cdot \mathrm{s}^{-1}\right)$ 。

\begin{tabular}{lllll}
\hline 方向 & 高度 & NW & NNW & NWW \\
\hline \multirow{2}{*}{$\alpha$} & $\leq 250 \mathrm{~m}$ & 0.42 & 0.41 & 0.50 \\
& $>250 \mathrm{~m}$ & 0.34 & 0.37 & 0.29 \\
\hline
\end{tabular}

由上可知, 不同来流方向 $250 \mathrm{~m}$ 以下区域地面粗鋉度 指数 $\alpha$ 的拟合值均大于更高高度的拟合值。而且, 拐点两 侧的地面粗糙度指数拟合值均大于我国建筑结构荷载规 范中D类地貌的对应值 $0.30[14]$ 。主要原因是观测场地位于 超大城市中心, 周边区域的建筑高度普遍较高导致的。其 中, NWW方向的 $\alpha$ 值在拐点两侧差异较大, 这是因为以 观测点为中心, 该方向较近区域的建筑高度较高, 而较远 区域建筑高度较低。

\section{3. 平均风向剖面}

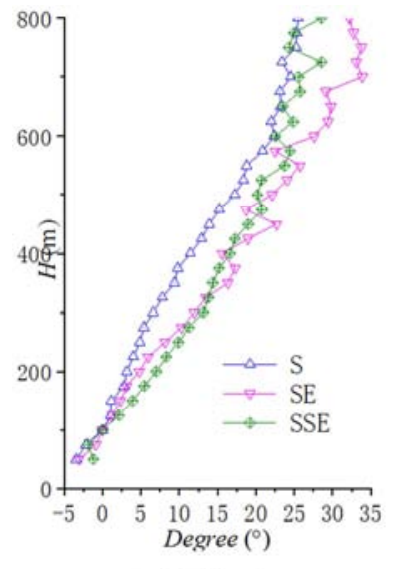

a 不同方向

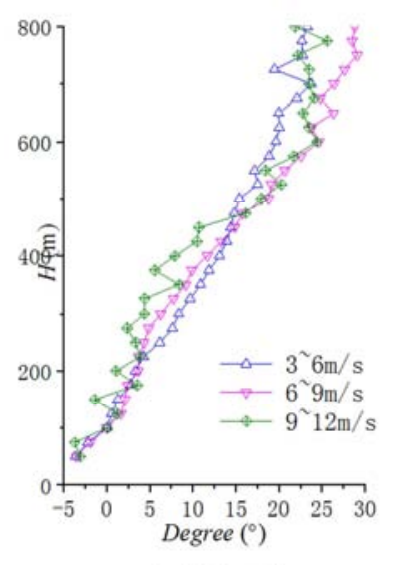

b 不同风速
图97月相对平均风向剖面。
对比分析7月和12月主要来流方向风向角相对于实测 $100 \mathrm{~m}$ 高度处风向的平均风向剖面图。其中所采用的样本 均以 $10 \mathrm{~min}$ 为基本时距进行处理, 并除去奇异点和坏点。 如图9、图10所示, 其中各条曲线均为同一风向下多个样 本数据的平均结果。

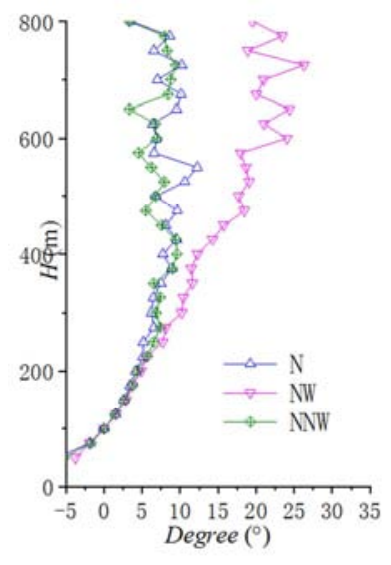

$\mathrm{a}$ 不同方向

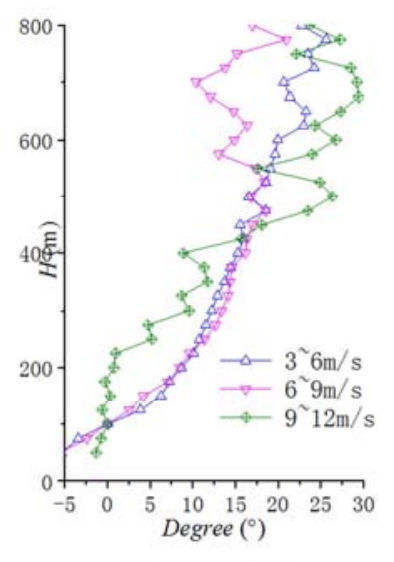

b 不同风速
图10 12月相对平均风向剖面。

7月和 12 月的三个主要来流方向的风向角沿高度的变 化如图9a、图10a所示。相对于 $100 \mathrm{~m}$ 高度处风向角的变化 均随着高度的提高而增大。由图中可见均表现为顺时针方 向的转动, 且从 $50 \mathrm{~m}$ 到 $1000 \mathrm{~m}$ 高度范围内的最大转角约为 $30^{\circ}$, 符合Ekman螺线的偏转规律。

但夏季和冬季又有所不同, 7月份主要风向S、SSE和 $\mathrm{SE}$ 的剖面曲线大体重合且变化平稳, 50 600m大气边界层 内偏转角度约为 $25^{\circ}$; 而 12 月份主要风向的剖面曲线在 $300 \mathrm{~m}$ 以上高度表现出较大差异, 其中风向 $\mathrm{N}$ 和 $\mathrm{NNW}$ 的样 本随高度增大偏转角度不再增大, 有较为明显的拐点, 风 向NW偏转角度则随高度增大继续增大, 符合Ekman螺线 特征。各风向剖面在 $600 \mathrm{~m}$ 以上高度变化不够稳定, 应当 是冬季阴雨等恶劣天气较多, 云层较厚, 影响了激光雷达 的测量精度。

图9b和图 $10 \mathrm{~b}$ 分别是是 7 月 $\mathrm{S}$ 方向和 12 月 $\mathrm{NW}$ 方向上不 同风速区间下的相对平均风向剖面。可见，夏季风向剖面 曲线仍然要比冬季稳定; 高度较低区域风向剖面曲线比高 度较高区域稳定; 风速较小区间的风向剖面曲线, 由于有 更多有效样本的支持, 要比风速较大区间稳定。Flay指出, 受地转偏向力和周边建筑物的影响, 近地层不同风速下同 一高度的风向存在着差异 $[15]$ 。

\section{4. 结论}

基于Windcube 100S多普勒激光测风雷达实测数据, 分析了上海城市中心上空的夏季和冬季良态风特性, 包括 风速风向时程、平均风速剖面、地面粗粘度指数、平均风 向剖面等, 得到以下结论:

（1）在大气边界层范围内, 平均风速随高度增大而 增加。空间上看, 各高度处的风速风向时程均呈现出很强 
的脉动性; 时间上看, 在同一季节, 平均风速的变化不是 很明显, 冬季风速会比夏季呈现更强的脉动性。

（2）上海市7月来流风向以南风和东南风为主， 12 月来流风向以西北风为主, 夏季相比于冬季, 来流更加集 中于主要方向, 风向更为稳定。

（3）在风速较大区间, 梯度风高度更大, 其平均风 速剖面曲线也更加符合指数率特征的规律性。冬季风剖面 梯度风高度要低于夏季。

（4）随着风速增加, 地面粗䊁度指数逐渐增大, 并 在12 15 m $\cdot \mathrm{s}^{-1}$ 高风速区间逐渐达到稳定。不同风向、不同 季节所拟合出的地面粗䊁度指数无明显差别。

(5) $250 \mathrm{~m}$ 以下高度不同方向的地面粗䊁度指数拟合 值均大于同方向 $250 \mathrm{~m}$ 以上高度的对应值, 且均大于 $\mathrm{D}$ 类地 貌下荷载规范值, 最大方向对应值可达 0.50 。

（6）不同方向上风向剖面变化有较高一致性，即偏 转角随高度递增, 在 $1000 \mathrm{~m}$ 高度内偏转角度约为 $30^{\circ}$, 符合 Ekman螺线规律。但冬季风向剖面曲线有较为明显的拐点, 而夏季风向剖面曲线的变化基本呈线性。

（7）夏季风向剖面曲线比冬季稳定; 高度较低区域 风向剖面曲线比高度较高区域稳定; 风速较小区间的风向 剖面曲线, 要比风速较大区间稳定。

\section{致谢}

本文为国家自然科学基金面上项目(51778493)，土木 工程防灾国家重点实验室自主课题 (SLDRCE19-B-13) 阶 段性成果之一。

\section{参考文献}

[1] Durst C S. Wind speeds over short periods of time [J]. The Meteorological Magazine, 1960, 89(1956):181-186.

[2] 傅抱璞,于静明.南京 164 米铁塔观测风速廓线的研究 [J]. 南 京大学学报(自然科学版), 1981(4):552-561。
[3] Cook N J. The Deaves and Harris. ABL model applied to heterogeneous terrain[J]. Journal of Wind Engineering and Industrial Aerodynamics, 1997,66: 197-214.

[4] 王澈泉,李正农, 胡佳星,张学文,周利芬, 曹守坤.城市地貌高 空台风特性及湍流积分尺度的研究 [J]. 空气动力学学 报,2017,35(06):801-806+822。

[5] 申建红.强风作用下高层建筑风场实测及模态参数识别研 究[D].上海大学, 2010 。

[6] 何浩博.超高层建筑施工期的风场特性与风振响应特性实 测研究[D].天津：天津大学,2016: 50-63。

[7] 顾明,匡军,全涌,等.上海环球金融中心大楼顶部风速实测数 据分析[J].振动与冲击, 2009,28(012):114-118。

[8] Li Q S, Fu J Y, Xiao Y Q, et al. Wind tunnel and full-scale study of wind effects on China's tallest building [J]. Engineering structures, 2006, 28(12):1745-1758.

[9] Rothermel J, Olivier L, Banta R, et al. Remote sensing of multi-level wind fields with highenergy airborne scanning coherent Doppler lidar. [J]. Optics Express, 1998, 2(2):40-50.

[10] 王乔乔, 张秀芝.Windcube激光雷达与测风塔测风结果对比 [J].气象科技.2013.01.004:1671-6345。

[11] 陈雯超,宋丽莉.不同天气条件下脉冲激光风廓线仪测风性 能 [J].应用气象学报, 2017,28(3):1001-7313。

[12] Tamura Y, Suda K, Sasaki A, et al. Simultaneous measurements of wind speed profiles at two sites using Doppler sodars [J]. Journal of wind engineering and industrial aerodynamics, 2001, 89 (3): 325-335.

[13] NJ Cook. The Designer's Guide to Wind Loading of Building Structures, [J]. Journal of Constructional Steel Research, 1985, 17 (3): 259-260.

[14] 中华人民共和国国家标准, 建筑结构荷载规范 [M], GB50009-2012,中华人民共和国住房和城乡建设部, 中华人 民共和国国家质量监督检验检疫总局, 中国建筑工业出版 社,2012:30-62。

[15] Flay R G J, Stevenson D C. Integral length scales in strong winds below $20 \mathrm{~m}[\mathrm{~J}]$. Journal of Wind Engineering and Industrial Aerodynamics, 1984, 28: 21-30. 